\title{
Development and validation of a prognostic nomogram for patients with triple-negative breast cancer with histology of infiltrating duct carcinoma
}

\author{
Na Jing ${ }^{1 \#}$, Ming-Wei Ma ${ }^{2 \#}$, Xian-Shu Gao ${ }^{2}$, Jian-Ting Liu ${ }^{1}$, Xiao-Bin Gu ${ }^{3}$, Min Zhang ${ }^{2}$, Bo Zhao ${ }^{4,5}$, \\ Yu Wang ${ }^{1}$, Xian-Ling Wang ${ }^{1}$, Hai-Xia Jia ${ }^{1}$ \\ ${ }^{1}$ Department of Radiation Oncology, Shanxi Cancer Hospital and the Affiliated Cancer Hospital of Shanxi Medical University, Taiyuan, China; \\ ${ }^{2}$ Department of Radiation Oncology, Peking University First Hospital, Beijing, China; ${ }^{3}$ Department of Radiation Oncology, The First Affiliated \\ Hospital of Zhengzhou University, Zhengzhou, China; ${ }^{4}$ Department of Engineering Physics, Tsinghua University, Beijing, China; Key Laboratory \\ of Particle \& Radiation Imaging, Ministry of Education (Tsinghua University), Beijing, China \\ Contributions: (I) Conception and design: N Jing, MW Ma; (II) Administrative support: XS Gao; (III) Provision of study materials or patients: MW \\ Ma, N Jing, JT Liu; (IV) Collection and assembly of data: N Jing, XB Gu, M Zhang, B Zhao, XL Wang, HX Jia; (V) Data analysis and interpretation: \\ MW Ma, Y Wang; (VI) Manuscript writing: All authors; (VII) Final approval of manuscript: All authors. \\ \#These authors contributed equally to this work. \\ Correspondence to: Xian-Shu Gao. Department of Radiation Oncology, Peking University First Hospital, No. 7 Xishiku Street, Beijing, China. \\ Email: doctorgaoxs@126.com.
}

Background: The purpose of this study was to develop prognostic nomograms from a cohort of patients with triple-negative breast cancer (TNBC) with histology of infiltrating duct carcinoma (IDC) by correlating their clinical and pathological parameters with the rates of disease-free survival (DFS) and overall survival (OS). Methods: We retrospectively analyzed TNBC patients with histology of IDC at our institution between 2009 and 2012. Age, family history, menopausal status, surgery type, T stage, N stage, histological grade, vascular invasion, perineural invasion, cytokeratin 5/6 status, Ki-67 expression, and epithelial cadherin (E-cadherin) status were analyzed. Predictors were used in multivariable logistic regression analysis to develop a nomogram to predict DFS and OS rates. The nomograms were then subjected to internal validation, with external validation of the nomogram for predicting OS using separate cohorts of TNBC patients known from the Cancer Genome Atlas (TCGA) database. Using the concordance index (C-index) with calibration curves, the predictive accuracy and discriminative ability were calculated.

Results: A total of 242 eligible TNBC patients were included for analysis. The median follow-up time was 70.73 months. Of the patients, $32.6 \%, 42.6 \%$, and $24.8 \%$ had stage I, II, and III disease, respectively. The 3- and 5-year survival rates were $81.0 \%$ and $76.5 \%$ for DFS, and $86.5 \%$ and $81.1 \%$, for OS, respectively. Age, $\mathrm{T}$ stage, $\mathrm{N}$ stage, and $\mathrm{E}$-cadherin status were found to be risk factors. The nomograms based on those risk factors accurately predicted the 3-and 5-year survival rates. The C-index was 0.798 and 0.821 for DFS and OS, respectively. Besides, the nomogram for OS showed relatively reliable performance in stratifying different risk groups of patients in training and validation cohorts identified from the TCGA database. The C-index reached 0.843 . DFS validation was not completed, as there was insufficient data.

Conclusions: Using clinicopathological information, we produced a prognostic nomogram that accurately predicts the 3- and 5-year DFS and OS for patients with TNBC with histology of IDC. More external confirmation is required.

Keywords: Triple-negative breast cancer (TNBC); disease-free survival (DFS); overall survival (OS); nomogram; comprehensive therapy

Submitted Jan 08, 2020. Accepted for publication Aug 07, 2020.

doi: $10.21037 /$ atm-20-413

View this article at: http://dx.doi.org/10.21037/atm-20-413 


\section{Introduction}

Triple-negative breast cancer (TNBC) accounts for $12-17 \%$ of all breast cancer (1) and is characterized as an absence in estrogen receptor (ER), progesterone (PR), and human epidermal growth factor receptor 2 (HER2) (2) immunohistochemical expression .TNBC presents as a heterogeneous group of tumors that is associated with a higher risk of the early development of local recurrence and visceral metastasis compared to other breast cancer subtypes (3). TNBC can be divided into various subtypes according to gene expression profiles (4), with a variable prognosis for each subtype. However, there is presently no consensus on the classification of the TNBC subtype. It is difficult to predict the prognosis of TNBC according to each subtype due to the technical complexity and expense. However, patient information including age, family history, menopausal status, operative type, and histological data such as tumor size are readily available for the prognostication of TNBC. Infiltrating duct carcinoma (IDC) is the most common histology among TNBC patients, accounting for $90 \%$ of breast cancer. Therefore, the development of a simple tool that uses the above data to predict the prognosis of TNBC patients with IDC histology could be clinically useful.

Nomograms are used as a prognostic device in all medical fields. The visual format of nomograms can provide a statistical predictive model that is readily understood by both patients and their physicians (5). One of the primary advantages of nomograms is their ability to estimate individualized risk based on patient and disease characteristics.

This study aimed to identify the prognostic factors for the disease-free survival (DFS) and overall survival (OS) of stage I-III TNBC patients with histology of IDC based on clinicopathological data and to develop easily applicable prognostic nomograms for the estimation of outcomes. This is the first research to establish nomograms based on the specific clinicopathological features of TNBC, to the best of our knowledge. We present the following article in accordance with the STROBE reporting checklist (available at http://dx.doi.org/10.21037/atm-20-413).

\section{Methods}

\section{Patients}

This retrospective study was conducted at Shanxi Cancer Hospital and the Affiliated Cancer Hospital of Shanxi Medical University between April 2009 and 2012, on a primary cohort of TNBC patients. The study was conducted in accordance with the Declaration of Helsinki (as revised in 2013). All study participants gave written informed consent, and the study was approved by ethics board of Shanxi Cancer Hospital and the Affiliated Cancer Hospital of Shanxi Medical University (No. 202035). We also verified that all procedures were carried out in compliance with the applicable guidelines and regulations. Inclusion criteria included the following: (I) patients who were histopathologically proven to have infiltrative, non-specific TNBC; (II) patients who had complete clinicopathological data; (III) patients who received a modified-radical mastectomy or breast-conserving surgery and postoperative chemotherapy and radiotherapy, according to the current National Comprehensive Cancer Network (NCCN) guidelines. Patients with in situ carcinoma or infiltrative specific carcinoma (non-infiltrating duct cancer) were excluded. Patients without standard post-operative treatment were also excluded. ER and PR negativity were defined as $<1 \%$ of cells staining positive, according to the American Society of Clinical Oncology/College of American Pathologists (ASCO/CAP) guidelines (6); HER2 negativity was scored as 0 or $1+$ in terms of its staining intensity by immunohistochemistry; if the score was $2+$, fluorescence in situ hybridization was required to confirm HER2 negativity. Positive membrane staining of epithelial cadherin (E-cadherin) was characterized as weak or strong. Cytokeratin 5/6 (CK5/6) positivity was defined as weak or strong cytoplasmic staining. The datasets that were used and/or analyzed during the current study are available from the corresponding author on reasonable request.

\section{Patient follow-up}

All patients were followed-up by hospitalization, outpatient visits, or telephone consultation. DFS and OS were used as the primary study endpoint and defined as the interval between the date of surgery and date of disease recurrence (any location) and death or the last date of follow-up.

\section{Validation of the developed nomogram}

To perform the external validation, we found 96 TNBC patients with stage T1-3N0-3M0 disease from the Cancer Genome Atlas (TCGA) acquired from the CBioPortal (http://www.cbioportal.org). We utilized the CDH1 gene, which encodes the mRNA expression level of cadherin 1, type 1, E-cadherin (epithelial) (CDH1) gene, as the 
expression data of E-cadherin. RNAseq read count below 10000 was defined as a group of lower expressions. Package TCGA-Biolinks $\mathrm{R}$ was used to extract clinical data and mRNA expression from TCGA datasets. Thus, enough information on the validation cohort was acquired to score all variables in the nomogram.

\section{Statistical analysis}

DFS and OS were estimated using the Kaplan-Meier method and used for univariate analysis. The factors with $\mathrm{P}<0.1$ were incorporated into a Cox proportional hazards regression model for multivariate survival analysis to evaluate individual prognostic factors. The predictors that were assessed in this investigation included age, family history, menopausal status, type of surgery (modified radical mastectomy versus breast-conserving), T stage, histological grade (divided as grade I-II, and III), N stage, vascular invasion, perineural invasion, cytokeratin 5/6 status, Ki-67 expression, and E-cadherin status.

Nomograms were constructed that were based on the clinically relevant factors and statistically significant factors derived from DFS and OS analysis of the Cox proportionalhazard regression. The performance of the nomograms was measured by the concordance index (C-index), and the calibration curves were calculated from the multivariate logistic model. Bootstrapping with 1000 resamples was used for these analyses. All the statistical analysis was performed using the open-source R statistical software ( $R$ Development Core Team 2008, Vienna, Austria).

\section{Results}

\section{Patient clinicopathologic characteristics}

A total of 242 patients met the criteria for inclusion. Table 1 lists the baseline characteristics of the primary TNBC patients with IDC histology who were analyzed in this study. The median patient age was 51 years (range, 29-69 years), and $67.4 \%$ of the patients had stage II-III disease. Only one patient had a grade 1 histological grade. The most common surgical technique was a modified-radical mastectomy $(n=222,91.7 \%)$.

\section{Kaplan-Meier evaluation of DFS and OS}

The median follow-up time was 70.73 months (range, 7.20-95.93 months). The 3- and 5-year survival rates were
Table 1 Clinical and pathologic characteristics of patients (n=242)

\begin{tabular}{|c|c|}
\hline Characteristic & Patients, n (\%) \\
\hline \multicolumn{2}{|l|}{ Age, y } \\
\hline Median (range) & 51 [29-69] \\
\hline$<40$ & $44(18.2)$ \\
\hline$\geq 40$ & $198(81.8)$ \\
\hline \multicolumn{2}{|l|}{ Family history } \\
\hline Yes & $47(19.4)$ \\
\hline No & $195(80.6)$ \\
\hline \multicolumn{2}{|l|}{ Menopausal status } \\
\hline Yes & $96(39.7)$ \\
\hline No & $146(60.3)$ \\
\hline \multicolumn{2}{|l|}{ Type of surgery } \\
\hline Modified radical mastectomy & $222(91.7)$ \\
\hline Breast-conserving surgery & $20(8.3)$ \\
\hline \multicolumn{2}{|l|}{ T stage } \\
\hline $\mathrm{T} 1$ & $110(45.5)$ \\
\hline $\mathrm{T} 2$ & $109(45.0)$ \\
\hline T3 & $23(9.5)$ \\
\hline \multicolumn{2}{|l|}{$\mathrm{N}$ stage } \\
\hline No & $138(57.0)$ \\
\hline N1 & $55(22.7)$ \\
\hline N2 & $32(13.2)$ \\
\hline N3 & $17(7.0)$ \\
\hline \multicolumn{2}{|l|}{ TNM stage } \\
\hline I & $79(32.6)$ \\
\hline II & $103(42.6)$ \\
\hline III & $60(24.8)$ \\
\hline \multicolumn{2}{|l|}{ Histological grade } \\
\hline 1 & $1(0.4)$ \\
\hline II & $117(48.4)$ \\
\hline III & $124(51.2)$ \\
\hline \multicolumn{2}{|l|}{ Vascular invasion } \\
\hline Yes & $58(24.0)$ \\
\hline No & $184(76.0)$ \\
\hline \multicolumn{2}{|l|}{ Perineural invasion } \\
\hline Yes & $3(1.2)$ \\
\hline
\end{tabular}

Table 1 (continued) 
Table 1 (continued)

\begin{tabular}{lc}
\hline Characteristic & Patients, $\mathrm{n}(\%)$ \\
\hline No & $239(98.8)$ \\
E-cadherin & \\
Negative & $17(7.0)$ \\
Positive & $225(93.0)$ \\
CK5/6 & \\
Negative & $73(30.2)$ \\
Positive & $169(69.8)$ \\
Ki67 & \\
$<14 \%$ & $30(12.4)$ \\
$\geq 14 \%$ & $212(87.6)$ \\
\hline
\end{tabular}

$81.0 \%$ and $76.5 \%$ for DFS, and $86.5 \%$ and $81.1 \%$ for OS, respectively. Of the 242 study patients, 50 (20.7\%) died during the follow-up period. Kaplan-Meier plots of DFS and OS for TNBC patients grouped according to age, T stage, histological grade, $\mathrm{N}$ stage, and E-cadherin status are shown in Figures 1 and 2.

\section{Independent prognostic factors}

In the univariate analysis, the prognostic factors with $\mathrm{P}$ value $<0.1$ in the cohort were as follows: age, $T$ stage, $\mathrm{N}$ stage, histological grade, E-cadherin status for both DFS and OS; and menopausal status, for DFS. See Table S1 for more information. The multivariate analysis found that $\mathrm{N}$ stage $(\mathrm{P}<0.001$ for both DFS and OS) and E-cadherin expression status $(\mathrm{P}<0.001$ for $\mathrm{DFS} ; \mathrm{P}=0.029$ for $\mathrm{OS}$ ) were independent risk factors for both DFS and OS. Age ( $\mathrm{P}=0.009$ for OS) and $\mathrm{T}$ stage $(\mathrm{P}=0.039$ for $\mathrm{OS})$ were independent risk factors for OS (Table 2). Since age and $\mathrm{T}$ stage have clinical significance, we incorporated all these four factors into the following nomograms for DFS and OS.

\section{Prognostic nomogram for DFS and OS}

Figure 3 illustrates the prognostic nomogram for the 3-and 5 -year DFS (A) and OS (B), as generated by the factors in the primary cohort. The calibration plots for the probability of DFS and OS showed a relatively high level of consistency between the actual observed outcome and the outcome that was predicted by the nomogram in the internal validation.
The predicted accuracy for DFS and OS, as measured by the C-index, was 0.798 (Figures $4 A, B$ ) and 0.821 (Figures 4C,D).

\section{Validation of predictive accuracy of the nomogram for OS}

Ninety-six patients were found as the validation cohort from the TCGA database. In the validation cohort, the 3and 5 -year OS rates were $86.6 \%$ and $67.9 \%$, respectively. The nomogram created from this cohort of patients is shown in Figure S1. The C-index of the nomogram for predicting OS was 0.843 . The calibration curves for the probability of 3- and 5-year OS were shown in Figure S2. As data were not enough in terms of analyzing DFS in the TCGA database, we did not perform this validation.

\section{Discussion}

In this study, we developed a prognostic nomogram that was based on the clinicopathological features of 242 patients with TNBC with IDC histology. The nomograms were able to predict the 3- and 5-year DFS and OS accurately. TNBC is drawing increased attention because of its specific biological characteristics (7), which lead to a higher probability of relapse and shorter OS times than other types of breast cancer. The most important reason for this is that TNBC does not benefit from endocrine therapy or targeted molecular therapies, as no appropriate target exists for these patients. TNBC has thus become the focal point of medical research (8). TNBC patients are at a higher risk of recurrence, usually from distant lung, brain, or soft tissue metastasis (between the first and third years after their primary treatment (9). Various TNBC subtypes have been identified using gene expression profiles and the presence of biomarkers, and these may be useful in biomarker selection as well as novel subtype-specific target identification $(4,10)$. However, there is presently no consensus on the subtype classification of TNBC.

Furthermore, no subtype classification using the above information is available for accurately predicting prognosis. At present, commonly used prediction tests such as MammaPrint score, PAM50/risk of recurrence/Prosigna kit Oncotype DX assay have all been used to confirm ERpositive patients (11). The other possible way to define outcomes among TNBC patients is to extract information on immune cell and inflammatory infiltration, although the practice value may be limited $(12,13)$. Thus, to set up a simple tool such as a nomogram using clinical features to predict the 

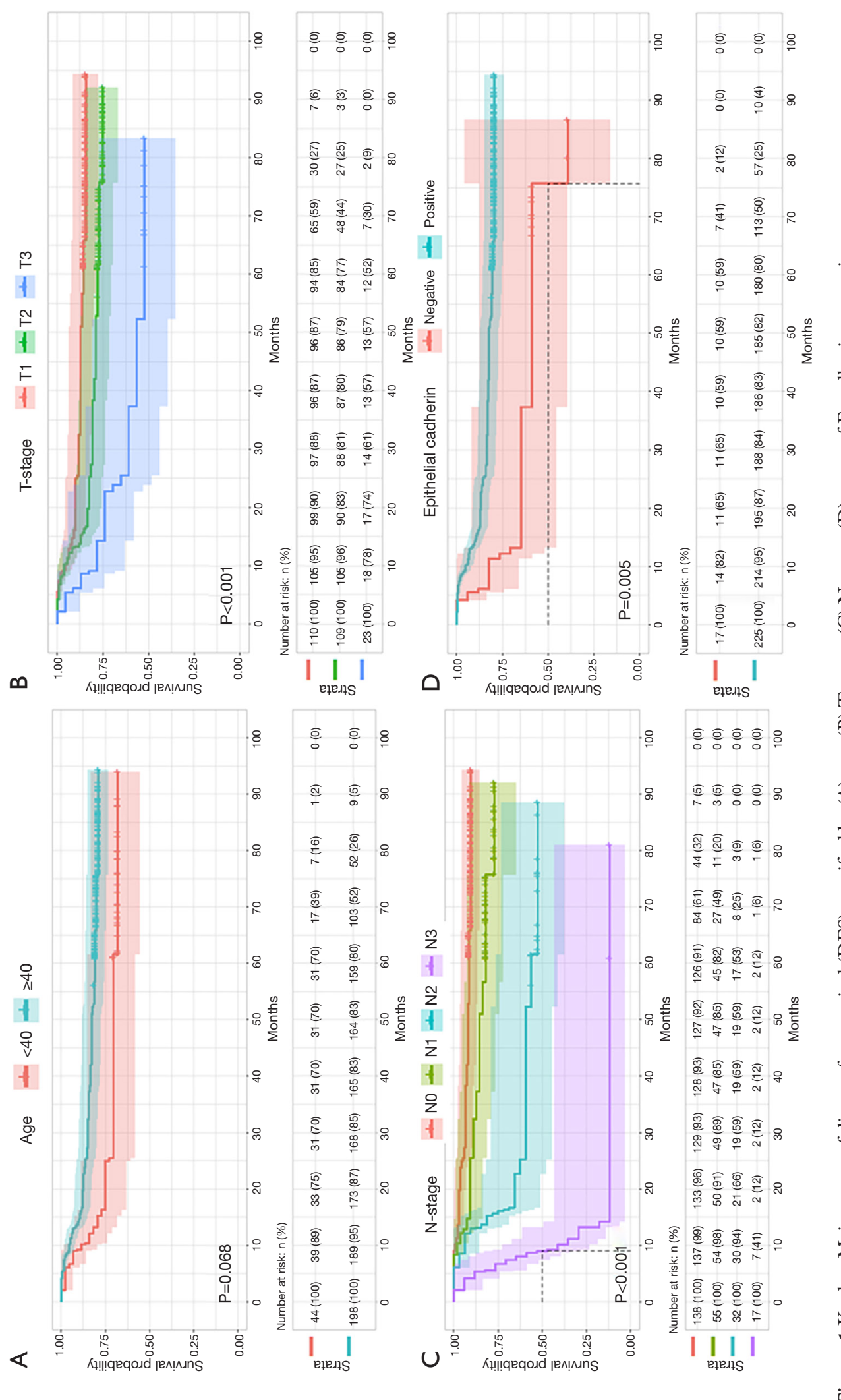


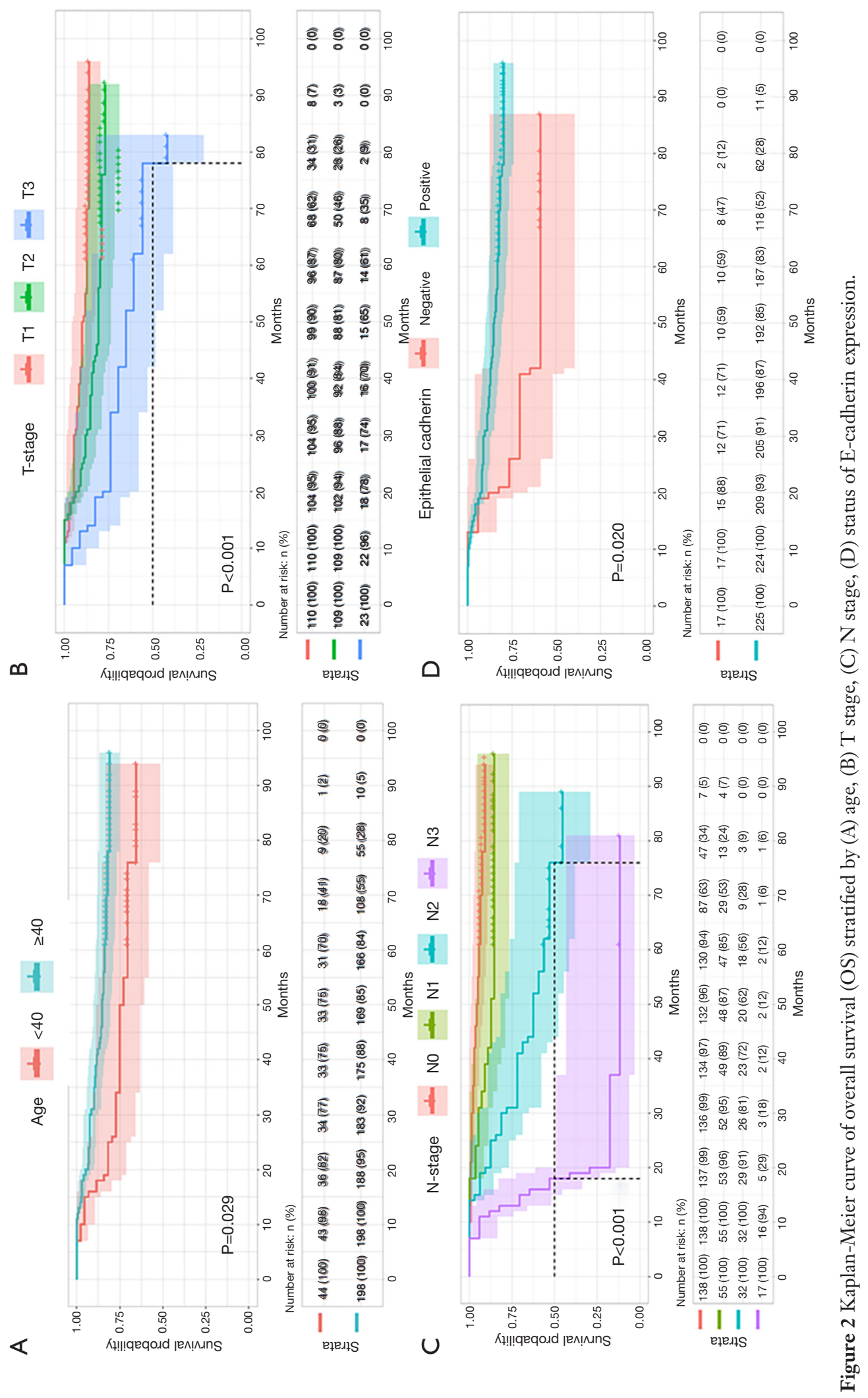


Table 2 Multivariate analyses for DFS and OS

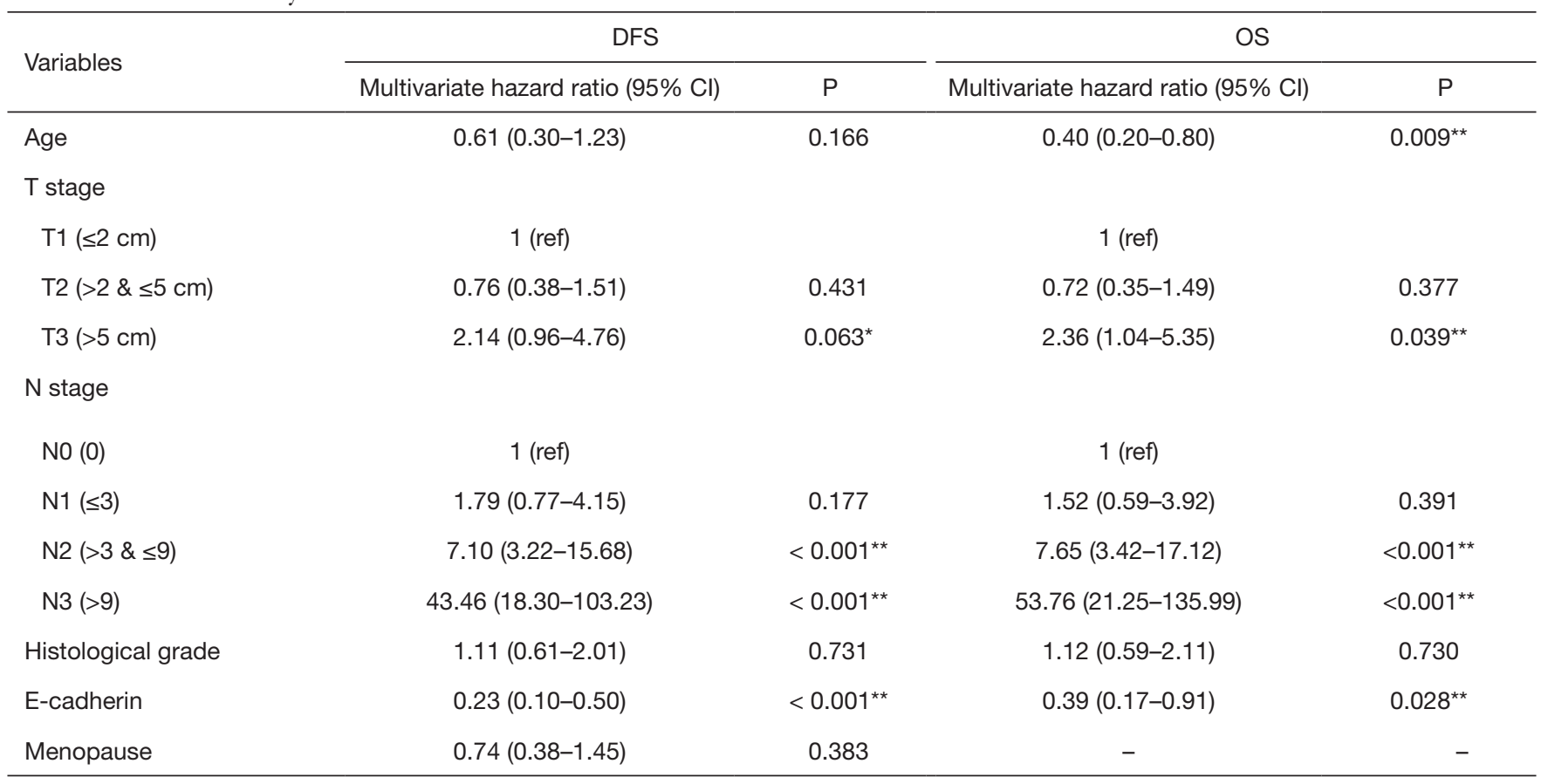

*, $\mathrm{P}<0.1 ;{ }^{* \star}, \mathrm{P}<0.05 . \mathrm{Cl}$, confidence interval.
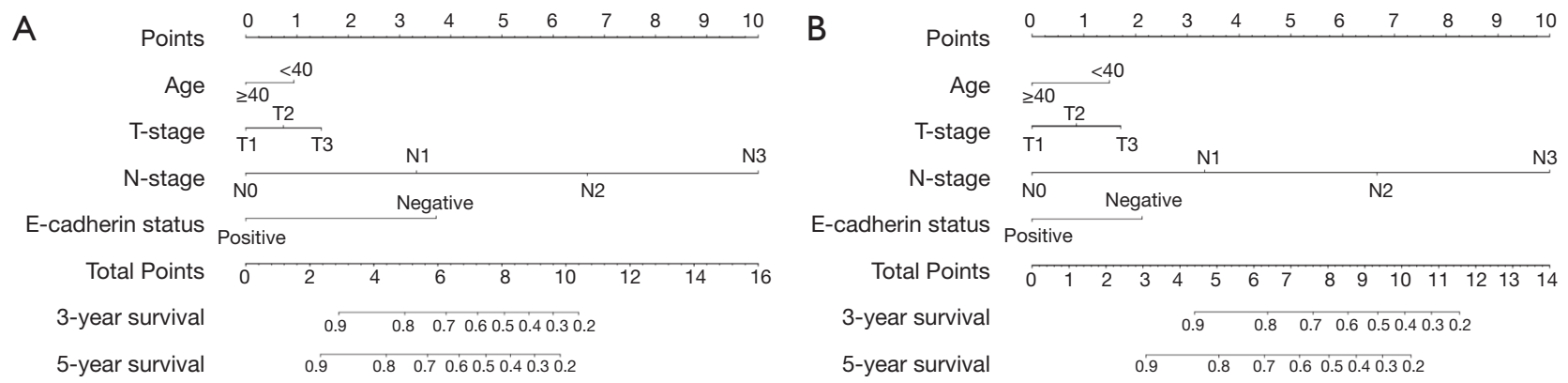

Figure 3 The nomograms that are used to predict the probability of DFS (A) and OS (B) for TNBC patients. Instructions: find the clinical parameters (e.g., age) on the respective axis and draw a line straight up to the point axis. Next, sum the points for each of the predictors and find the final sum on the total point axis. Lastly, draw a line straight down to find the patient's probability of DFS or OS.

prognosis of TNBC patients is reasonable in clinical practice.

A previous study (14) showed that the size of a tumor and the status of the axillary lymph node were independent prognostic factors. Additionally, TNBC patients are typically younger at diagnosis than patients with other types of breast cancer (15). As was stated in the previous study, ER-negative cancers tend to be poorly differentiated (16). Only one patient in our group had a grade I tumor. After categorizing the group into grade I-II and grade III, the histological classification showed no significance, which may be explained by the small sample size. E-cadherin is encoded by $C D H 1$, which is a member of the classical cadherins class and is a vascular endothelial cadherin. It plays a vital role in tissue formation. It is defined as a tumor suppressor gene that slows cancer cell progression because it mediates cell-cell communication at the basolateral membrane in adherent junctions, and it also plays a crucial role in epithelial-to-mesenchymal transition (EMT) (17). In our study, the absence of E-cadherin indicated a decrease in DFS and OS, and another study (18) has also found that it is an important independent prognostic factor. Age, $\mathrm{T}$ stage, $\mathrm{N}$ stage, and the status of $\mathrm{E}$-cadherin expression were found 

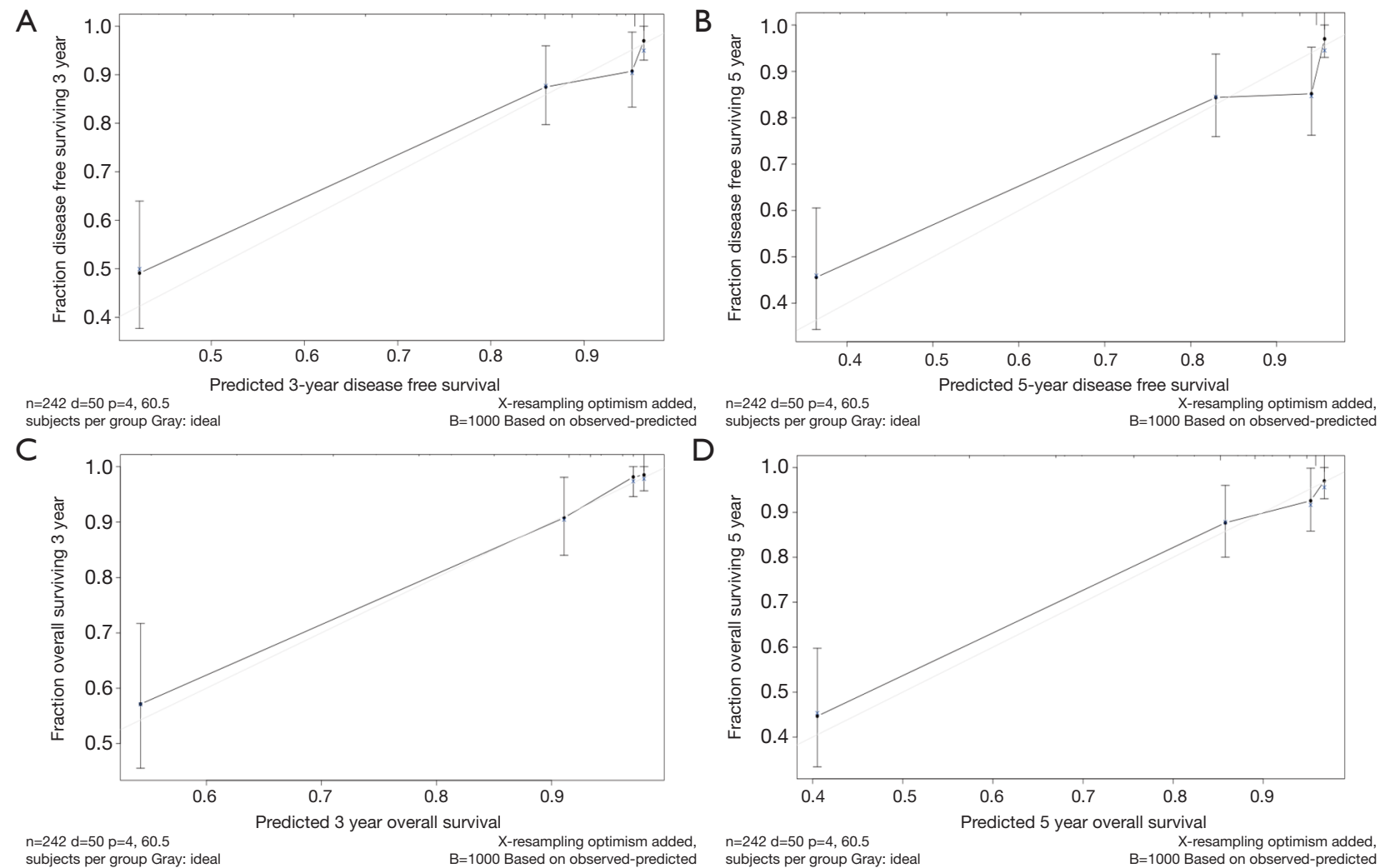

Figure 4 Calibration curves of the nomograms for (A) predicting the 3-year and (B) 5-year DFS, and (C) 3-year and (D) 5-year OS in the primary cohort, respectively.

to have significant prognostic value in our study.

Nomograms are currently used to measure cancerspecific survival (19), overall survival (20), and the value of adjuvant therapy (21) in breast cancer patients. To date, several studies have predicted OS in TNBC patients and presented powerful models for breast cancer care. Dai (22) used data from the Surveillance, Epidemiology, and End Results (SEER) program to construct a model that predicts three-year OS. However, since only $10.9 \%$ of the patients died, the follow-up time was short. Another study predicted the pathologic complete response of neoadjuvant chemotherapy, which is applicable for treatment decisionmaking (23). Other nomograms for TNBC patients established through the TCGA database included one for patients with non-infiltrating ductal carcinoma, which is an independent prognostic factor (13).

In our study, we generated a predictive tool for TNBC with IDC histology patients at their first disease presentation and pathological diagnosis that had high accuracy. Most of the patients in our study had stage II-III disease. The 3- and 5 -year DFS and OS rates in our cohort are consistent with the results of other recent studies $(24,25)$. In the primary cohort, our model integrating the above features was based on the clinically relevant factors of the Cox proportional hazards model and coefficients and showed good discrimination. Although the sample size of the validation cohort of TCGA datasets was small, the results of the calibration curves suggested that this nomogram was useful for predicting the short-term overall survival of TNBC patients. A larger sample size of the validation sets was required for the longterm survival results.

As TNBC stands out for its aggressive behavior, new biomarkers (26) and treatment entities for controlling TNBC are being explored, and many promising results have been found. In a prospective, multicenter study which investigated the addition of pembrolizumab to neoadjuvant chemotherapy for early TNBC patients, a significantly higher pathological complete response $(64.8 \%)$ was found in the pembrolizumab-chemotherapy group as compared with the placebo-chemotherapy group (51.2\%) (27). Another study designed and validated novel nitrogen-based chalcone analogs, which could induce a reversal of EMT 
by upregulating the E-cadherin (28). Using this model, we may use hypothesize that it is desperately important for patients with lower DFS or OS to try to incorporate new modalities other than traditional therapy to manage the disease. For instance, a 45-year-old patient with TNBC with IDC histology, who had a tumor of $4 \mathrm{~cm}$ in size, one positive axillary lymph nodes, and negative E-cadherin expression may have a probability of $82 \%$ and $72 \%$ for OS and $55 \%$ and $50 \%$ for DFS at 3 and 5 years after diagnosis, respectively. That is to say, even though the patient is in a relatively early stage, there would be half the risk for them to suffer tumor progression five years after the surgery. More aggressive or newer agents for her was needed.

This study is limited by its small sample size and retrospective, single-center nature; therefore, further external validation is required. Additionally, data of other pathological prognostic parameters such as epidermal growth factor receptor (EGFR), androgen receptor (AR), tumor-infiltrating lymphocytes (TILs), and the breast cancer susceptibility genes (BRCA) mutation that are potentially related to prognosis are lacking. The establishment and validation of nomograms that incorporate newly and specific markers in a large cohort of TNBC patients need to be pursued.

\section{Conclusions}

We generated a multifactorial nomogram that combines clinicopathological factors with classical TNBC with IDC histology. Based on this model, early aggressive therapy may be called for in the management of patients with poor prognosis. However, multicenter data and more prognostic factors are needed to confirm this nomogram for clinical practice.

\section{Acknowledgments}

Funding: This work was supported by Scientific Research Seed Fund of Peking University First Hospital [grant number 2018SF043 to MWM] and Scientific Research Project of Shanxi Municipal Commission of Health and Family Planning [grant number 2017075 to NJ] and Beijing Municipal Science and Technology Plan "Capital Featured Clinical Application Research" Special Project [grant number Z161100000516041 to MZ].

\section{Footnote}

Reporting Checklist: The authors have completed the
STROBE reporting checklist. Available at http://dx.doi. org/10.21037/atm-20-413

Data Sharing Statement: Available at http://dx.doi. org/10.21037/atm-20-413

Peer Review File: Available at http://dx.doi.org/10.21037/ atm-20-413

Conflicts of Interest: All authors have completed the ICMJE uniform disclosure form (available at http://dx.doi. org/10.21037/atm-20-413). The authors have no conflicts of interest to declare.

Ethical Statement: The authors are responsible for all aspects of the work in ensuring that questions related to the accuracy or integrity of any part of the work are appropriately investigated and resolved. The study protocol was approved by the Ethics Committee of Shanxi Cancer Hospital and the Affiliated Cancer Hospital of Shanxi Medical University (No. 202035), in accordance with the Declaration of Helsinki (as revised in 2013). Written informed consent was obtained from all patients or their surrogate when possible.

Open Access Statement: This is an Open Access article distributed in accordance with the Creative Commons Attribution-NonCommercial-NoDerivs 4.0 International License (CC BY-NC-ND 4.0), which permits the noncommercial replication and distribution of the article with the strict proviso that no changes or edits are made and the original work is properly cited (including links to both the formal publication through the relevant DOI and the license). See: https://creativecommons.org/licenses/by-nc-nd/4.0/.

\section{References}

1. Boyle P. Triple-negative breast cancer: epidemiological considerations and recommendations. Ann Oncol 2012;23:7-12.

2. Foulkes WD, Smith IE, Reis-Filho JS. Triple-negative breast cancer. N Engl J Med 2010;363:1938-48.

3. Lowery AJ, Kell MR, Glynn RW, et al. Locoregional recurrence after breast cancer surgery: a systematic review by receptor phenotype. Breast Cancer Res Treat 2012;133:831-41.

4. Burstein MD, Tsimelzon A, Poage GM, et al. Comprehensive genomic analysis identifies novel subtypes 


\section{Page 10 of 10}

and targets of triple-negative breast cancer. Clin Cancer Res 2015;21:1688-98.

5. Balachandran VP, Gonen M, Smith JJ, et al. Nomograms in oncology: more than meets the eye. Lancet Oncol 2015;16:e173-80.

6. Hammond ME, Hayes DF, Wolff AC, et al. American society of clinical oncology/college of american pathologists guideline recommendations for immunohistochemical testing of estrogen and progesterone receptors in breast cancer. J Oncol Pract 2010;6:195-7.

7. Lee JA, Kim KI, Bae JW, et al. Triple negative breast cancer in Korea-distinct biology with different impact of prognostic factors on survival. Breast Cancer Res Treat 2010;123:177-87.

8. Carey LA, Dees EC, Sawyer L, et al. The triple negative paradox: primary tumor chemosensitivity of breast cancer subtypes. Clin Cancer Res 2007;13:2329-34.

9. Lin NU, Vanderplas A, Hughes ME, et al. Clinicopathologic features, patterns of recurrence, and survival among women with triple-negative breast cancer in the National Comprehensive Cancer Network. Cancer 2012;118:5463-72.

10. Lehmann BD, Bauer JA, Chen X, et al. Identification of human triple-negative breast cancer subtypes and preclinical models for selection of targeted therapies. J Clin Invest 2011;121:2750-67.

11. Shachar SS, Muss HB. Internet tools to enhance breast cancer care. NPJ Breast Cancer 2016;2:16011.

12. Oner G, Altintas S, Canturk Z, et al. Triple-negative breast cancer-Role of immunology: A systemic review. Breast J 2020;26:995-9.

13. Wang K, Li HL, Xiong YF, et al. Development and validation of nomograms integrating immune-related genomic signatures with clinicopathologic features to improve prognosis and predictive value of triple-negative breast cancer: A gene expression-based retrospective study. Cancer Med 2019;8:686-700.

14. Wishart GC, Azzato EM, Greenberg DC, et al. PREDICT: a new UK prognostic model that predicts survival following surgery for invasive breast cancer. Breast Cancer Res 2010;12:R1.

15. Lund MJ, Trivers KF, Porter PL, et al. Race and triple negative threats to breast cancer survival: a populationbased study in Atlanta, GA. Breast Cancer Res Treat 2009;113:357-70.

16. Gyorffy B, Hatzis C, Sanft T, et al. Multigene prognostic tests in breast cancer: past, present, future. Breast Cancer Res 2015;17:11.
Jing et al. Nomogram for triple-negative breast cancer patients

17. Gall TM, Frampton AE. Gene of the month: E-cadherin (CDH1). J Clin Pathol 2013;66:928-32.

18. Shen T, Zhang K, Siegal GP, et al. Prognostic Value of E-Cadherin and beta-Catenin in Triple-Negative Breast Cancer. Am J Clin Pathol 2016;146:603-10.

19. Kattan MW, Gonen M, Jarnagin WR, et al. A nomogram for predicting disease-specific survival after hepatic resection for metastatic colorectal cancer. Ann Surg 2008;247:282-7.

20. Yang L, Takimoto T, Fujimoto J. Prognostic model for predicting overall survival in children and adolescents with rhabdomyosarcoma. BMC Cancer 2014;14:654.

21. Wang SJ, Patel SG, Shah JP, et al. An oral cavity carcinoma nomogram to predict benefit of adjuvant radiotherapy. JAMA Otolaryngol Head Neck Surg 2013;139:554-9.

22. Dai D, Jin H, Wang X. Nomogram for predicting survival in triple-negative breast cancer patients with histology of infiltrating duct carcinoma: a population-based study. Am J Cancer Res 2018;8:1576-85.

23. Zhang F, Huang M, Zhou H, et al. A Nomogram to Predict the Pathologic Complete Response of Neoadjuvant Chemotherapy in Triple-Negative Breast Cancer Based on Simple Laboratory Indicators. Ann Surg Oncol 2019;26:3912-9.

24. Kaplan HG, Malmgren JA. Impact of triple negative phenotype on breast cancer prognosis. Breast J 2008;14:456-63.

25. Ovcaricek T, Frkovic SG, Matos E, et al. Triple negative breast cancer - prognostic factors and survival. Radiol Oncol 2011;45:46-52.

26. da Silva JL, Cardoso Nunes NC, Izetti P, et al. Triple negative breast cancer: A thorough review of biomarkers. Crit Rev Oncol Hematol 2020;145:102855.

27. Schmid P, Cortes J, Pusztai L, et al. Pembrolizumab for Early Triple-Negative Breast Cancer. N Engl J Med 2020;382:810-21.

28. Elkhalifa D, Siddique AB, Qusa M, et al. Design, synthesis, and validation of novel nitrogen-based chalcone analogs against triple negative breast cancer. Eur J Med Chem 2020;187:111954.

Cite this article as: Jing N, Ma MW, Gao XS, Liu JT, Gu XB, Zhang M, Zhao B, Wang Y, Wang XL, Jia HX. Development and validation of a prognostic nomogram for patients with triple-negative breast cancer with histology of infiltrating duct carcinoma. Ann Transl Med 2020;8(21):1447. doi: 10.21037/atm$20-413$ 